\title{
1 Aberrant Activation of hsa-miR-181d/STAT3 and hsa- miR-181d/5A Ratios Mediate the Anticancer Effect of 3 Garcinol in STAT3/5A-Addicted GBM
}

4 Heng-Wei Liu 1,2,3,4, Peter Mingjui Lee 5, Oluwaseun Adebayo Bamodu 4,6,7, Yu-Kai Su 1,2,3,4, IatHang Fong ${ }^{3,4}$, Chi-Tai Yeh ${ }^{1,4,6,7}$, Ming-Hsien Chien ${ }^{1}$, I-Hung Kan ${ }^{1,2,3,4, *}$ and Chien-Min Lin ${ }^{1,2,3,4, *}$

${ }^{1}$ Graduate Institute of Clinical Medicine, College of Medicine, Taipei Medical University, Taipei City 11031, Taiwan; henryway0404@hotmail.com (H.-W.L.); mhchien1976@gmail.com (M.-H.C.) 2Department of Neurology, School of Medicine, College of Medicine, Taipei Medical University, Taipei City 11031, Taiwan. yukai.su@gmail.com (Y.-K.S.)

${ }^{3}$ Division of Neurosurgery, Department of Surgery, Taipei Medical University-Shuang Ho Hospital, New Taipei City 23561, Taiwan. nskan1999@icloud.com

${ }^{4}$ Taipei Neuroscience Institute, Taipei Medical University, Taipei 11031, Taiwan. m513092004@tmu.edu.tw 5California Northstate University College of Medicine, Elk Grove, California, CA 95757, United States of America (peter100893@gmail.com).

${ }^{6}$ Department of Hematology \& Oncology, Cancer Center, Taipei Medical University - Shuang Ho Hospital, New Taipei City, 235, Taiwan (16625@s.tmu.edu.tw)

7Department of Medical Research \& Education, Taipei Medical University - Shuang Ho Hospital, New Taipei City, 235, Taiwan (ctyeh@s.tmu.edu.tw)

* Correspondence:

Dr. I-Hung Kan, Division of Neurosurgery, Department of Surgery, Taipei Medical University-Shuang Ho Hospital, New Taipei City 23561, Taiwan. Tel: +886-2-2490088 ext. 8881, Fax: +886-2-2248-0900. E-mail: nskan1999@icloud.com

Dr. Chien-Min Lin, Taipei Neuroscience Institute, Taipei Medical University, Taipei 11031, Taiwan. m513092004@tmu.edu.tw

Abstract:

Background: Glioblastoma (GBM), a malignant grade IV tumor, is the most malignant brain tumor due to its hyper-proliferative and apoptosis-evading characteristics. The signal transducer and activators of transcription (STAT) family genes, including STAT3 and STAT5A, have been indicated to play important roles in GBM progression. Increasing number of reports suggest that Garcinol, a polyisoprenylated benzophenone and major bioactive component of Garcinia indica contains potent anti-cancer activities. Material \& Methods: The present study investigated the anti-GBM effects of garcinol, focusing on the STAT3/STAT5A activation, using a combination of bioinformatics, in vitro, and ex vivo assays. Results: Our bioinformatics analysis of TCGA - GBM cohort $(n=173)$ showed that STAT3 and STAT5A are preferentially elevated in primary and recurrent GBM, compared to nontumor brain tissues, and is significantly correlated with reduced overall survival. In support, our immunohistochemical staining of a GBM cohort $(n=30)$ showed an estimated 5.3-fold $(p<0.001)$ elevation in STAT3 and STAT5A protein expression in primary and recurrent GBM versus the nontumor group. In vitro, garcinol treatment significantly suppressed the proliferative, invasive, and migratory potential of U87MG or GBM8401 cells, dose-dependently. In addition, garcinol anticancer effect significantly attenuated the GBM stem cell-like phenotypes, as reflected by diminished ability of U87MG or GBM8401 to form colonies and tumorspheres and suppressed expression of OCT4 and SOX2. Furthermore, analysis on GBM transcriptome revealed an inverse correlation between the level of STAT3/5A and hsa-miR-181d. Garcinol-mediated anti-GBM effects were associated with an increased hsa-miR-181d/STAT3 and hsa-miR-181d/5A ratio. Conclusion: We present evidence of anti-GBM efficacy of garcinol mediated by enhancing the hsa-miR-181d/STAT3 and hsa-miR$181 \mathrm{~d} / 5 \mathrm{~A}$ ratios in GBM cells. Our findings suggest a potential new therapeutic agent for combating aggressive GBM.

Keywords: glioblastoma; GBM; glioma; STAT3; STAT5A; hsa-miR-181d; microRNA

Working title: Garcinol effectively inhibit glioblastoma 


\section{Introduction}

Glioblastoma (GBM), a WHO grade IV malignant glioma associated with poor prognosis, is characterized by enhanced cellularity, increased mitotic activity, vascular hyperproliferation and pseudopallisading necrosis (Adamson et al., 2009; Omuro \& DeAngelis, 2013). Being highly invasive, GBM cells infiltrate the surrounding brain parenchyma, including the cerebral cortex, cerebellum, brainstem, and the spinal cord (Adamson et al., 2009). Current treatment options for GBM, namely surgery, chemotherapy, and radiotherapy are characterized by dismal curative success, as evidenced by the limited increase in the median overall survival (OS) from 12 months to 15 months in patients exposed to radiation therapy and temozolomide (Omuro \& DeAngelis, 2013; Stupp et al., 2005). Symptomatic treatments such as corticosteroids for managing peritumoral edema, antiseizure drugs (ASDs) for reducing seizures, and the anti-angiogenesis monoclonal antibody, Bevacizumab (also known as Avastin), to mitigate brain edema are also used; howbeit, aside improving patients' quality of life (QoL), they confer no survival advantage on patients with GBM (Kreisl et al., 2009; Omuro \& DeAngelis, 2013; Rossetti \& Stupp, 2010). In addition, contemporary evidence indicates that the 1year-survival rate of patients with de novo GBM is $17-30 \%$, and the 2-year survival rate is set at a dismal 3-5\% (Adamson et al., 2009). This dearth of effective anti-GBM therapeutic strategy, against the background of dismal clinical outcome, necessitates urgent discovery of new actionable molecular targets and/or development of novel efficacious anti-GBM therapy.

The last 20 years has been characterized by accruing evidence of the role of cancer stem cells (CSCs, otherwise known as cancer-initiating cells) in enhanced oncogenicity and metastatic phenotype, resistance to therapy, cancer relapse, and consequently poor clinical outcome in patients with malignancies, including GBM (Colak \& Medema, 2014; Frank, Schatton, \& Frank, 2010; Jackson, Hassiotou, \& Nowak, 2015; Phi et al., 2018; Toledo-Guzmán, Bigoni-Ordóñez, Ibáñez Hernández, \& Ortiz-Sánchez, 2018). CSC transcription factors (TFs) are promising anti-cancer therapeutic targets. Contemporary literature is replete with documented association of the signal transducer and activator of transcription (STAT) proteins with enhanced pluripotency, related CSC-like phenotypes, and cancer progression (Cheng et al., 2018; Do et al., 2013; Matthews, Sansom, \& Clarke, 2011). STAT proteins are a family of TFs that facilitate numerous biological processes such as cell proliferation, differentiation, survival, and inflammation (Yue \& Turkson, 2009). The aberrant expression and/or activities of several members of the STAT protein family, including STAT3 and STAT5A, have been implicated in the initiation, growth, and metastatic dissemination of various cancers, including head and neck, breast and brain cancers (Guryanova et al., 2011). The aberrant expression of STAT3 has been implicated in GBM development and progression, and has been suggested to be a master regulator of the mesenchymal cum malignant transformation of gliomas (Carro, Maria stella; Lim, Wei; Alvarez, Mariano; Bollo, Robert; Zhao, Xudong; Snyder, Evan; Sulman, Erik; Anne, Sandrine; Doetsch, Fiona; Colman, Howard; Lasorella, Anna; Aldape, Ken; Califano, Andrea; Iavarone, 2010). $\mathrm{Li}$ et al. demonstrated that lentivirus-mediated silencing STAT3 in GBM cells induces cellular differentiation, indicating its role in keeping an undifferentiated cellular state in malignancy (G. Li et al., 2009). As with STAT3, STAT5A is a key effector of the Janus Tyrosine Kinase (JAK)/STAT pathway, its phosphorylation being positively correlated with cell invasion and poor prognosis in GBM (Cao et al., 2011; Latha et al., 2013; Roos et al., 2018). Fan et al. demonstrated that STAT5 signaling drives pro-tumorigenic phenotypes in GBM cells (Fan et al., 2013). Thus, in the light of all these evidences suggesting that STAT3 and STAT5A play critical roles in cancer progression, the present study investigated the nature and extent of the involvement of STAT3 and STAT5A in the therapeutic response and disease progression of patients with GBM.

Over the last 2 decades, there has been increase in the documentation of the critical roles of microRNA (miRNAs/miRs) in the malignantization and progression of many human malignancies, including GBM; by their ability to hamper or facilitate cancer initiation, miRNAs have emerged as therapeutically-relevant actionable biomolecules for anticancer therapy (Bhaskaran et al., 2019; Floyd \& Purow, 2014). One such miRNA family is miRNA-181, with varied expression of its isoforms (miRNA-181a/b/c/d) being touted as independent predictors of clinical outcome in patients with different cancer types (Pop-Bica et al., 2018). A notable feature of treatment-resistant GBM is the 
102

103

104

105

106

107

108

109

110

111

112

113

114

115

116

117

118

119

120

121

122

123

124

125

126

127

128

129

130

131

132

133

enzyme $\mathrm{O}^{6}$-methylguanin-DNA methyltransferase (MGMT), a DNA-repair protein that removes alkyl group from the $\mathrm{O}^{6}$ position of alkyl groups, and consequently diminish the curative effects of chemotherapeutic agents (Hegi et al., 2005), conversely, the silencing of MGMT through promoter methylation is associated with prolonged OS and disease-free survival (DFS) in patients with GBM (Bell et al., 2018). Interestingly, recent studies show that MGMT activity is inversely correlated with the expression of microRNA-181d/miRNA-181d; as higher expression of miRNA-181d is positively correlated with improved OS in patients with GBM (Zhang et al., 2012). There is also accruing evidence that the prognostic implications of altered miRNA expression, is connected to their roles in modulation of stemness signaling such as Notch, Hedgehog, and JAK/STAT3 (Floyd \& Purow, 2014; F. Yang et al., 2017), and the consequent acquisition of stem cell-like traits by GBM cells. Thus, the present study's rationale for exploring the actionability of miRNA-181d in the context of STAT signaling in GBM.

Due to the accruing adverse reactions to most chemotherapeutic agents, phytochemicals and nutraceuticals have garnered interest as possible safe alternatives or adjuvants in cancer treatment (Saadat \& Gupta, 2012). Building on previous works by our team demonstrating that Garcinol inhibits CSC-like phenotype by suppressing the Wnt/ $\beta$-catenin/STAT3 signaling axis in human non-small cell lung carcinoma (Huang et al., 2018), we now examined the probable effect of Garcinol on GBM stem cells (GBM-SCs), and the implication of same for sensitivity to conventional chemotherapy and better prognosis. Garcinol, a major bioactive constituent of the fruit Garcinia indica, has widely-documented antioxidant and anticancer effects, and is chemically similar in structure to the well-known curcumin (Ashad et al., 2017; Saadat \& Gupta, 2012). In fact, Hong et al. (Hong et al., 2007) demonstrated that garcinol significantly inhibited the growth of the colon cancer cells, and importantly, provided evidence that unlike conventional chemotherapeutics, garcinol preferentially targets cancerous cells; thus inhibiting cancer growth without adversely affecting the neighboring 'normal' non-cancerous cells (Hong et al., 2007). In breast cancer, garcinol was shown to inhibit STAT3-NF-kB signaling, resulting in reduced invasiveness, in vitro, and significantly attenuated tumor growth in NOD-SCID mice (Ahmad et al., 2012, 2010); thus, building a case for the preclinical investigation of the probable anti-GBM effect of garcinol in this stance. This present study, for the first time, to the best of our knowledge, investigated and documents the effect of garcinol on GBM, consistent with current theme-relevant knowledge, especially in the context of the therapeutic effects of garcinol alone or in synergism with conventional anticancer treatment modalities on GBM-SCs through the mediation of STAT3/5A signaling and miRNA-181d.

\section{Methods}

\subsection{Drugs and Chemicals}

Garcinol (sc-200891A, HPLC purity $\geq 95 \%$ ) purchased from Santa Cruz Biotechnology (Santa Cruz, CA, USA) was dissolved in dimethyl sulfoxide (DMSO) to prepare a $20 \mathrm{mM}$ stock and stored at $-20^{\circ} \mathrm{C}$ until use. For different assays, the stock was further diluted using cell growth medium as appropriate. Dimethyl sulfoxide (DMSO), served as vehicle and negative control. Unless otherwise indicated, all reagents were obtained from Gibco (Thermo Fisher Scientific, Life Technologies, Foster City, CA, USA).

\subsection{Analyses of Cancer RNAseq Dataset}

The Cancer Genome Atlas (TCGA) GDC-TCGA glioblastoma (GBM) cohort ( $n=173)$ used for STAT3 and STAT5A gene expression profiling and correlative studies, was accessed, downloaded and analyzed using the University of California Santa Cruz (UCSC) Xena functional genomics explorer platform (https://xenabrowser.net/heatmap/\#) .

\subsection{Cell lines and Culture}


The human U-87 MG (ATCC $®$ HTB-14 ${ }^{\mathrm{TM}}$ ) (ATCC, Manassas, VA) and GBM8401 GBM cell lines used in the study were purchased from (Bioresource Collection Research Center, Hsinchu, Taiwan). The cell lines were cultured in Gibco DMEM (CAT. No. 11965175, Thermo Fisher Scientific, Inc. Waltham, MA, USA), supplemented with $10 \%$ fetal bovine serum (FBS) and $1 \%$ penicillin/streptomycin (Invitrogen, Life Technologies, Carlsbad, CA, USA) and incubated in 5\% humidified $\mathrm{CO}_{2}$ incubator at $37^{\circ} \mathrm{C}$. The cells were sub-cultured when they reached $80-90 \%$ confluency and the media changed every $48-72 \mathrm{~h}$.

\subsection{Sulforhodamine B (SRB) Viability Assay}

GBM8401 and U87MG cells were seeded in 96-well plates in triplicates at a concentration of 3.5 $x 10^{3}$ cells per well. After $24 \mathrm{~h}$ incubation in a $5 \% \mathrm{CO} 2$ humidified incubator at $37^{\circ} \mathrm{C}$, the cells were treated with varying concentrations of $2.5 \mu \mathrm{M}-40 \mu \mathrm{M}$ garcinol as indicated for $24 \mathrm{~h}$. Thereafter, cells were washed in cold PBS, fixed in 10\% trichloroacetic acid (TCA) for $1 \mathrm{~h}$, washed with distilled water, and then incubated in 0.4 SRB $(w / v)$ in $1 \%$ acetic acid at room temperature for $1 \mathrm{~h}$. After washing of unbond SRB dye with 1\% acetic acid thrice, the plates were air-dried, and bond SRB dye dissolved in $20 \mathrm{mM}$ Tris base under gentle agitation for $5 \mathrm{~min}$ and the absorbance was read at $570 \mathrm{~nm}$ wavelength in a microplate reader (Molecular Devices, Sunnyvale, CA, USA).

\subsection{Western Blot Analysis}

U87MG and GBM8401 cells pre-treated with or without $2.5 \mu \mathrm{M}$ or $5 \mu \mathrm{M}$ of garcinol for $24 \mathrm{~h}$ were lysed, and the cellular protein lysates extracted using the Protein Extraction Kit (QIAGEN, Germantown, MD, USA), followed by quantification using the Bradford Protein Assay Kit. $20 \mu \mathrm{g}$ protein samples were then loaded per lane and protein separated in $10 \%$ sodium dodecylsulfate polyacrylamide gel electrophoresis (SDS-PAGE) gels, blots were transferred onto polyvinylidene fluoride (PVDF) membranes, and then non-specific binding blocked in 5\% skimmed milk in Tris-buffered saline with Tween20 (TBST) for 1h. Thereafter, blot-bearing PVDF membranes were incubated overnight at $4{ }^{\circ} \mathrm{C}$ with the following primary antibodies: p-STAT3 (\#9145; 1:1000 dilution), STAT3 (\#9132; 1:1000 dilution), p-ERK (\#4370; 1:1000), ERK1/2 (\#4695; 1:1000), p-Akt1/2/3 (\#4060; 1:1000), Akt1/2/3 (\#2920, 1:1000), Bax (\#5023; 1:1000), Bcl-xl (\#2764; 1:1000) from Cell Signaling Technology (cell signaling, MA, USA), p-STAT5 (ab32364; 1:1000), STAT5 (ab227687; 1:1000 dilution) from Abcam (Abcam, MA, USA), GAPDH (10494-1-AP; 1:10000) from Proteintech Group (Proteintech, IL, USA). Please see the Supplementary Table 1. This was followed by washing membranes thrice in TBST for $5 \mathrm{~min}$ each, before incubition with appropriate goat anti-mouse or antirabbit horseradish peroxidase (HRP)-linked secondary antibodies for $1 \mathrm{~h}$ and TBST washing three times for $5 \mathrm{~min}$ each. The protein bands were visualized using enhanced chemiluminescence (ECL) detection system (ECL, Amersham Pharmacia Biotech, NJ, USA), and quantified using ImageJ software (https://imagej.nih.gov/ij/).

\subsection{Immunohistochemical (IHC) Staining}

The study was approved by the Joint Institutional Review Board (JIRB) of the Taipei Medical University -Shuang Ho Hospital (Approval number: N201903047). Tissue samples from patients with primary and recurrent GBM were obtained from the Taipei Medical University-Shuang Ho Hospital GBM cohort $(\mathrm{n}=45)$. After de-waxing the paraffin-embedded $4 \mu \mathrm{m}$ tissue sections using xylene for $5 \mathrm{~min}$ twice and re-hydrating with 100\% ethanol twice for $5 \mathrm{~min}, 95 \%$ ethanol for $5 \mathrm{~min}$ and $80 \%$ ethanol for $5 \mathrm{~min}, 3 \%$ hydrogen peroxide $\left(\mathrm{H}_{2} \mathrm{O}_{2}\right)$ (TA-125- $\mathrm{H} 2 \mathrm{O} 2 \mathrm{Q}$, Thermo Fisher Scientific, Waltham, MA, USA) was used to block endogenous peroxidase activity for $10 \mathrm{~min}$. The sections were then immersed in $10 \mathrm{mmol} / \mathrm{L}$ ethylenediaminetetraacetic acid (EDTA) for $3 \mathrm{~min}$ in a pressure cooker, then blocked with $10 \%$ normal serum. Thereafter, tissue samples were incubated with primary antibody against STAT3 $(1: 200)$ or STAT5 $(1: 200)$ at $4{ }^{\circ} \mathrm{C}$ overnight, and then with biotin-labeled secondary antibody at room temperature for $1 \mathrm{~h}$. Sections were incubated in diaminobenzidine (DAB) and then counterstained with hematoxylin. Visualization was done under a light microscope. 


\subsection{Immunofluorescence Staining and Quantification}

U87MG and GBM8401 cell lines pre-treated with or without $2.5 \mu \mathrm{M}$ or $5 \mu \mathrm{M}$ of garcinol were seeded in 6-well chamber slides (Nunc, Thermo Fisher Scientific, Taipei, Taiwan), incubated at $4^{\circ} \mathrm{C}$ overnight, fixed with $2 \%$ paraformaldehyde for $10 \mathrm{~min}$ at room temperature, and permeabilized in $0.01 \mathrm{M}$ phosphate-buffered saline (PBS) with $0.1 \%$ Triton $\mathrm{X}-100$ and $0.2 \%$ bovine serum albumin (BSA). The slides were air-dried and rehydrated with PBS before incubation with primary antibodies against OCT4 (\#2840; Cell Signaling Technology) and/or SOX2 (\#3579; Cell Signaling Technology) at 1:500 dilution in PBS for $2 \mathrm{~h}$ at room temperature. The slides were washed with PBS twice for $10 \mathrm{~min}$ each, then incubated with anti-rabbit IgG fluorescein isothiocyanate (FITC)-conjugated secondary antibody (diluted 1:500; Jackson Immunoresearch Lab. Inc., West Grove, PA, USA) in PBS for 1h. The slides were mounted with Vectashield mounting medium and counter stained with 4', 6'-diamidino2-phenylindole (DAPI) for nucleus visualization.

\subsection{Tumorsphere Formation Assay}

For tumorsphere formation, U87MG and GBM8401 cells were cultured in Chemicon ${ }^{\circledR}$ serumfree HEScGRO medium for human embryonic stem cell culture (CAT. No. SCM020, Merck KGaA, Darmstadt, Germany) supplemented with $10 \mathrm{ng} / \mathrm{mL}$ human recombinant basic fibroblast growth factor (hbFGF; Invitrogen, Carlsbad, CA, USA), $20 \mathrm{ng} / \mathrm{mL}$ human epithelial growth factor (hEGF; Millipore, Bedford, MA, USA), B27 supplement (Invitrogen, Carlsbad, CA, USA), heparin (CAT. No. 07980; STEMCELL Technologies Inc., Interlab Co., Ltd, Taipei, Taiwan), and NeuroCult ${ }^{\mathrm{TM}}$ NS-A proliferation supplement (CAT. No. 05753, STEMCELL Technologies Inc., Interlab Co., Ltd, Taipei, Taiwan). The cells were seeded at a concentration of $1 \times 10^{3} \mathrm{cell} / \mathrm{s} / \mathrm{mL} /$ well in 6-well ultra-low adhesion plates (Corning Inc., Corning, NY, USA) with or without $5 \mu \mathrm{M}$ of garcinol and incubated at $37^{\circ} \mathrm{C}$ in $5 \%$ humidified $\mathrm{CO}_{2}$ atmosphere for $7-10$ days. The anchorage-independent tumorspheres $(\geq 90 \mu \mathrm{m}$ in diameter) were photographed under inverted phase contrast microscope.

\subsection{Colony Formation Assay}

U87MG and GBM8401 cells were seeded in triplicates at a density of $2 \times 10^{4}$ cells per well in 6well culture plates (Corning, Corning, NY, USA) with complete growth media containing $0 \mu \mathrm{M}, 2.5$ $\mu \mathrm{M}$, or $5 \mu \mathrm{M}$ of garcinol and incubated at $37^{\circ} \mathrm{C}$ for $12-14$ days. Culture plates with colonies with $\geq 100$ $\mu \mathrm{m}$ in diameter and $\geq 50$ cells were washed with PBS twice, fixed with methanol for $15 \mathrm{~min}$, and stained with $0.005 \%$ crystal violet for $15 \mathrm{~min}$ at room temperature. The colonies formed were then visualized under microscope and counted using the ChemiDoc-XRS imager (QuantityOne software package; Bio-Rad, Hercules, CA, USA)

\subsection{Invasion Assay}

$3 \times 10^{4}$ U87MG and GBM8401 cells were seeded per well onto $8 \mu \mathrm{m}$ pore membrane coated with Matrigel in the upper chamber of 24-well Transwell chambers containing serum-free DMEM medium with $0,2.5$, or $5 \mu \mathrm{M}$ of garcinol, while the lower chambers contained growth media with $10 \%$ FBS serving as chemo-attractant. After $24 \mathrm{~h}$ incubation the media was discarded, and the non-invaded cells on the upper surface of the insert were removed with sterile cotton swabs, while the invaded GBM cells on lower surface of the filter membrane were fixed with $3.7 \%$ formaldehyde for $1 \mathrm{~h}$, and stained with crystal violet dye for $20 \mathrm{~min}$. The stained cells were visualized under microscope and then analyzed using NIH ImageJ software (https://imagej.nih.gov/ij/).

\subsection{Wound-healing migration Assay}

After U87MG and GBM8401 cells were seeded in 24-well plates (Corning, Corning, NY, USA) with DMEM with $10 \%$ FBS and incubated until 100\% confluency, scratch-wounds were made along the median axis of the adherent monolayer cells using sterile $200 \mu \mathrm{L}$ micropipette tips. The wells were carefully washed with PBS to remove detached cells and then incubated in new growth media 
containing $0,2.5$, or $5 \mu \mathrm{M}$ of garcinol for $24 \mathrm{~h}$ or $48 \mathrm{~h}$. Photographs of scratch-wound healing were taken at indicated time-point.

\subsection{Real-time polymerase chain reaction ( $q R T-P C R)$ Analysis}

Total RNA extracted from U87MG or GBM8401 cells treated with or without $5 \mu \mathrm{M}$ of garcinol using Trizol reagent (Invitrogen, Carlsbad, CA, USA) following manufacturer's instruction. $2 \mu \mathrm{g}$ of RNA was added to the real time PCR, with the final primer concentration being $0.5 \mu \mathrm{M}$. The PCR was performed under the following condition: reverse transcription at $42^{\circ} \mathrm{C}$ for $60 \mathrm{~min}$, amplification for 30 cycles at $94^{\circ} \mathrm{C}$ for $30 \mathrm{sec}, 58^{\circ} \mathrm{C}$ for $50 \mathrm{sec}$, and $72^{\circ} \mathrm{C}$ for $50 \mathrm{sec}$.

\subsection{Statistical Analysis}

All experiments were performed at least 3 times in triplicates. Data presented represent means \pm SD of all results. The comparison between two groups was done using 2-sided Student's $t$-test, while one-way analysis of variance (ANOVA) was used to compare $\geq 3$ groups. A $p$-value $<0.05$ was considered statistically significant.

\section{Results}

3.1. STAT-3 and STAT-5 are highly expressed in primary and recurrent glioblastoma, and their expression negatively correlates with overall survival rates

Firstly, against the background that STAT3 and STAT5 to be highly expressed in GBM cell lines (Roos et al., 2018), we evaluated the expression profiles of STAT proteins GDC TCGA-GBM cohort of 173 samples. Our results showed a 1.07-fold or 1.08-fold increase in the median gene expression of STAT3 in the primary and recurrent GBM samples, respectively, compared to non-tumor samples; similarly, compared to the non-tumor control, the median expression of STAT5A was elevated by 1.08-fold or 1.09-fold the primary and recurrent GBM samples, respectively (Figure 1A). In line with the above, our survival analyses showed a significantly strong association between high STAT3 $(p<$ $0.015)$ or STAT5 ( $\mathrm{p}$ 0.008) expression levels and worse OS (Figure 1B, upper). Interestingly, we also demonstrated that compared with the $\sim 20 \%, 11 \%$, or $10 \%$ OS amongst patients with STAT3 lowSTAT5A ${ }^{\text {low }}$ STAT3 lowSTAT5A ${ }^{\text {high }}$, or STAT3 ${ }^{\text {high }}$ STAT5A ${ }^{\text {low }}$ GBM, respectively, no patient with STAT3 ${ }^{\text {highSTAT5A }} \mathrm{A}^{\text {high }}$ GBM was alive, suggesting a strong association between STAT3 ${ }^{\text {high STAT5 }} \mathrm{A}^{\text {high }}$ and GBM-specific mortality $(\mathrm{p}<0.007)$ (Figure 1B, lower). Moreover, consistent with the RNA expression, results of our immunohistochemical staining confirmed elevated STAT3 and STAT5A protein expression levels in primary and recurrent GBM tissues compared to non-tumor tissues (STAT3/5A vs non-tumor: 5.3-fold, $\mathrm{p}<0.001$ ) (Figures 1C and 1D). These results indicate, at least in part, that enhanced STAT3 and STAT5A expression and/or activity play a critical role in the development and recurrence of GBM.

\subsection{Garcinol significantly inhibits GBM cell viability and oncogenicity through induction of STAT3/5A signaling and enhanced apoptosis}

Against the background of recent work demonstrating that garcinol inhibits CSC-like phenotype of human non-small cell lung carcinoma by suppressing the Wnt/ $\beta$-catenin/STAT3 signaling axis (Huang et al., 2018), we investigated the probable STAT signaling-mediated anti-GBM effect of garcinol (Figure 2A). Firstly, to provide some mechanistic insight, we demonstrated that treatment of U87MG or GBM8401 cells with $2.5 \mu \mathrm{M}$ or $5 \mu \mathrm{M}$ garcinol significantly downregulated the expression of p-STAT3, p-STAT5, p-ERK, and p-AKT (Figure 2B), Synchronous with the observed inhibition of STAT3, STAT5 and AKT signaling, garcinol significantly suppressed the viability of GBM4801 and U87MG cells, with $10 \mu \mathrm{M}$ eliciting $51 \%$ or $25 \%$ reduced viability of U87MG or GBM8401 cells, respectively, and $40 \mu \mathrm{M}$ eliciting $94.7 \%$ reduction of U87MG and GBM8401 cell viability, indicating a dose-dependent GBM cell killing effect (Figure 2C), and this reduced viability 
was associated with markedly enhanced Bax/Bcl-xL apoptotic ratio, as $2.5 \mu \mathrm{M}$ induced a 1.67-fold $(\mathrm{p}<0.05)$ or 2.7 -fold $(\mathrm{p}<0.05)$ increase in U87MG or GBM8401 apoptotic ratio, while $5 \mu \mathrm{M}$ increased the apoptotic ratio by 2.83 -fold $(\mathrm{p}<0.001)$ or 2.92 -fold $(\mathrm{p}<0.001)$ in the U87MG or GBM8401 cells, respectively (Figure 2D). Since the highly invasive GBM spreads fast to surrounding brain tissue, thus, contributing to its documented lethality (Omuro \& DeAngelis, 2013), we sought to understand if and how garcinol affects this invasive trait. We demonstrated that treatment with 2.5 $\mu \mathrm{M}$ or $5 \mu \mathrm{M}$ dose-dependently suppressed the migration of the U87MG $(\sim 59 \%, \mathrm{p}<0.01$ or $81 \%, \mathrm{p}<$ 0.001 , respectively) and GBM8401 ( 48\%, p $<0.01$ or $76 \%$, p $<0.001$, respectively) cells at the $24 \mathrm{~h}$ timepoint (Figure 2E). Similarly, $2.5 \mu \mathrm{M}$ or $5 \mu \mathrm{M}$ garcinol induced a $60 \%(\mathrm{p}<0.01)$ or $\sim 80 \%(\mathrm{p}<0.001)$ reduction of U87MG invasive capacity, respectively, and 39\% (p<0.01) or $60 \%(\mathrm{p}<0.001)$ reduction in number of invaded GBM8401 cells (Figure 2F). Together, these data suggest that garcinol significantly inhibits GBM cell viability and oncogenicity through induction of STAT3/5A and associated signaling with enhanced apoptosis.

\subsection{Garcinol negatively impacts GBM stem cell-like phenotypes}

Understanding that the highly prevalent and malignant GBM harbors self-renewing, tumorigenic GBM-SCs that facilitate tumor initiation and resistance to therapy (Lathia et al., 2015; Beier, Schulz, \& Beier, 2011). To assess the effects of garcinol on GBM-SCs, we performed tumorsphere and colony formation assays on the GBM8401 and U87MG cell lines. The results from the tumorsphere assay demonstrated that $5 \mu \mathrm{M}$ garcinol significantly caused both cell lines to lose their ability to form GBM tumorspheres, quantitatively and qualitatively, with $\sim 88 \%$ ( $\mathrm{p}<0.01$ ) reduction in the number of U87MG or GBM8401 tumorspheres formed, and $~ 96 \%(\mathrm{p}<0.01)$ or $89 \%$ $(\mathrm{p}<0.001)$ reduction in the U87MG or GBM8401 tumorsphere sizes, respectively (Figure 3A). Furthermore, because of the biological relevance of clonality in GBM-SCs origin (Lathia et al., 2015), we demonstrated that $2.5 \mu \mathrm{M}-5 \mu \mathrm{M}$ garcinol significantly inhibited the ability of the GBM cells to form colonies, dose-dependently, as $2.5 \mu \mathrm{M}$ reduced the number of formed U87MG or GBM8401 colonies by $49 \%(\mathrm{p}<0.05)$ or $36 \%(\mathrm{p}<0.05)$, respectively, while $5 \mu \mathrm{M}$ induced a $75 \%(\mathrm{p}<0.01)$ or $72 \%$ $(p<0.01)$ reduction, respectively (Figure $3 \mathbf{B})$. Contextually, the garcinol-induced inhibition of tumorsphere and colony formation potential was associated with significant and dose-dependent down-regulation of the nuclear expression of stemness proteins SOX2 and OCT4 (Figure 3C) as shown with immunofluorescence assay, and this inhibitory effect was associated with significantly suppressed STAT3 and STAT5A immunofluorescence, in a dose-dependent manner (Figure 3D). These data are indicative of the negative influence of garcinol on the stem cell-like phenotypes of GBM cells.

\subsection{Garcinol increases the expression of hsa-miR181d, which has inhibitory effects on STAT3 and STAT5 expression}

Having established that garcinol impairs STAT3 and STAT5A activation, we probed for likely modulators and/or mediators of the interaction between garcinol and the STAT proteins. Hsa-miR181d shown in Figure 4A, has been implicated in the worse OS of patients with GBM (Zhang et al., 2012). Consistent with this, we demonstrated that hsa-miR-181d binds with STAT3 with a mirSVR or PhastCons score of -0.26 or 0.69 , respectively, while it binds with STAT5A with a mirSVR or PhastCons score of -0.21 or 0.49 , respectively (Figure $4 \mathrm{~A}$ ). Where the mirSVR predicted the likelihood of hsa-miR-181d down-regulating the target mRNA STAT3 and STAT5A based on the sequence and structure features in the miRNA/mRNA predicted target sites. Moreover, the PhastCons score showed the likelihood that the predicted miRNA/mRNA binding nucleotides are conserved. Next, our qRT-PCR analysis of $5 \mu \mathrm{M}$ garcinol-treated U87MG and GBM8401 cells showed that garcinol significantly induced higher expression of miR-181d in the U87MG (2.7-fold, $p<0.01)$ and GBM8401 (2.1-fold, $\mathrm{p}<0.01$ ) cells (Figure 4B). To confirm a direct relationship between the STAT proteins and miR-181d expression, western blot analysis was done comparing samples exposed to mir-181d inhibitor, mir-181d-mimic, or mir-181d inhibitor/garcinol combination. The results show that mir-181d inhibitor significantly enhanced the expression of p-STAT3 and pSTAT5 proteins 
compared to the control group, while for the mir-181d-mimic-treated cells, the p-STAT3 and pSTAT5 protein expression levels were significantly lower, and for cells incubated with mir-181d inhibitor and $5 \mu \mathrm{M}$ garcinol concomitantly, p-STAT3 and p-STAT5 protein expression levels were markedly higher than in the mir-181d-mimic group but lower than the mir-181d-inhibitor group (Figure 6C). These results indicate that garcinol can activate mir-181d activity which suppresses STAT3/5A activation.

\section{Discussion}

STAT3 and STAT5 are transcription factors implicated in various tumor cell proliferation, migration, and invasion (Roos et al., 2018). STAT3 and STAT5A in response to cytokines and growth factors are activated by phosphorylation of their Y705 position and Y695 residues, respectively, followed by nuclear translocation of the phosphorylated proteins with subsequent activation of specific downstream gene transcription (Mohanty et al., 2017). Activation of STAT3 and STAT5 serve different purposes. STAT3 has been shown to promote the proliferation and pathobiology of GBM tumor cores, while STAT5 affects its local invasion capabilities (Roos et al., 2018). Evidence also abound that STAT3 signaling plays a role in maintaining the self-renewal capabilities and multilineage differentiation potential of glioma stem cells (GSMs) (Guryanova et al., 2011). As with STAT3, genetic alterations in the STAT5A gene has been implicated in myeloproliferative disorders and linked to hematopoietic stem cell proliferation (Ghanem et al., 2017).

Past works from our team demonstrated that garcinol inhibited STAT3 activation and suppressed the lung cancer stem cell population (Huang et al., 2018). In the present study, investigating if glioblastoma patients also exhibit high levels of STAT3 and the associated STAT5A, we performed RNAseq analysis of the GDC-TCGA glioblastoma cohort and demonstrated that both primary and recurrent glioblastoma patients have higher expression of STAT3 and STAT5. Consistent with past evidence showing aberrant STAT activity in glioblastoma cell lines, immunohistochemical analysis revealed amplified STAT3/5A expression in primary and recurrent GBM. The increased RNA and protein expression indicated STAT3 and STAT5A play a critical role in driving GBM tumorigenesis. Furthermore, recurrent glioblastoma displayed a general trend of increased STAT5A expression, suggesting a specific role for STAT5A in maintaining the GBM-SC phenotype. Upon analysis of the differential STAT3/5A expression-based survival rates, high expression of both STAT3 and STAT5A was found to be strongly associated with the poorest OS time; and may be connected with the enhanced tumorigenicity and lethality of GBM in oncology clinics. Of translational relevance, we observed no significant difference in OS time between the STAT3 ${ }^{\text {highSTAT5 }} \mathrm{A}^{\text {low }}$ cohort and the STAT3lowSTAT5A high cohort, suggesting there is no specific advantage in inhibiting the expression of either transcription factor, and highlighting probable therapeutic efficacy of parallel inhibition of both STAT3 and STAT5A. Thus, as indicated by the dismal OS of patients with

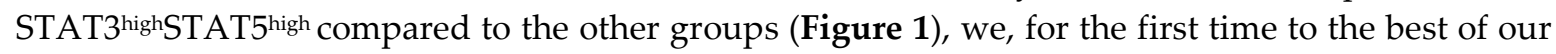
knowledge demonstrate that concerted targeting of the aberrant expression and/or activity of STAT3/5A in GBM, both at protein and mRNA levels, is essential for any meaningful curative effect in STAT-based targeted therapy for patients with primary or even recurrent GBM. This would be consistent with recent evidence indicating that combined targeting of STAT3 and STAT5 effectively reversed various forms of tyrosine kinase inhibitor (TKI)- resistance in highly resistant BCRABL1 ${ }^{\text {T315I }}$ chronic myeloid lymphoma (CML) cells (Gleixner et al., 2016).

In the context of the GBM SC-phenotypes including enhanced proliferation, oncogenicity, therapy resistance, recurrence and poor prognosis, we further demonstrated that garcinol significantly inhibited GBM8401 and U87MG cell viability in a dose-dependent manner, as well as suppresses the cell invasive and migratory potentials, thus, demonstrating the anti-proliferative and anti-metastatic efficacy of garcinol in GBM (Figure 2). This is consistent with documented robust growth-inhibitory effects of garcinol demonstrated against colon cancer and immortalized intestinal cells (Hong et al., 2007). Since STAT3 and STAT5A are implicated in the maintenance of the stem celllike characteristics of GBM, we examined garcinol's therapeutic effect on the GBM-SCs profile. Interestingly, low-dose garcinol $(\leq 5 \mu \mathrm{M})$ deregulated STAT3/5A signaling with repressed AKT and 
ERK crosstalk, and this was sufficient to significantly impeded GBM cell migration, invasion, clonogenicity, and tumorsphere formation, with associated increase in apoptotic index and nuclear expression of SOX2 and OCT4 (Figures 3 and 4). Our findings are corroborated by recent evidence that STAT3 and STAT5 are constitutively activated in malignant cells, and that their persistent activation facilitates cancer development and progression by altering downstream gene expression through epigenetic modification, EMT induction, oncogenic modification of the tumor microenvironment, and enhancing of CSCs self-renewal and differentiation (Yuan et al., 2015), as well as evidence implicating high ERK1/2 activity in the acquisition and maintenance of SOX2-expressing Glioma stem cells (Kwon et al., 2017).

\section{Conclusion}

Taken together, as depicted in our pictorial abstract (Figure 5), the present study provides evidence that the constitutive activation of STAT3/5A in GBM is inversely correlated with suppressed has-miR-181d expression, and that garcinol-induced upregulation of hsa-miR-181d/STAT3 and hsamiR-181d/5A ratios mediate the anti-GBM-SC effect of garcinol in STAT3/5A-addicted GBM. These findings are of translational relevance as they highlight the therapeutic efficacy of a relatively novel small molecule inhibitor of STAT3/5A in the highly invasive and often therapy-resistant GBM

Funding: This work was supported by National Science Council of Taiwan: Chien-Min Lin (MOST 107-2314-B-038 -056 -MY3) and grants to Yu-Kai Su (MOST 107-2314-B-038-022 -). This study was also supported by grants from Taipei Medical University, Taiwan (106-FRP-03) to Chien-Min Lin.

Authors' contribution: Study conception and experimental design: Heng-Wei Liu, Peter Mingjui Lee. Performed the experiments: Oluwaseun Adebayo Bamodu, Iat-Hang Fong. Data collation and analysis: Yu-Kai Su. and Chi-Tai Yeh. Manuscript writing: Oluwaseun Adebayo Bamodu, Peter Mingjui Lee. Provided reagents, materials, and experimental infrastructure: Chi-Tai Yeh, Ming-Hsien Chien, I-Hung Kan and Chien-Min Lin. All authors read and approved the final submitted version of the manuscript.

Ethics approval and consent to participate: The study was approved by the Joint Institutional Review Board (JIRB) of the Taipei Medical University -Shuang Ho Hospital (Approval number: N201903047). Tissue samples from patients with primary and recurrent GBM were obtained from the Taipei Medical University-Shuang Ho Hospital GBM cohort $(n=45)$.

Availability of data and materials: The datasets used and analyzed in the current study are publiclyaccessible as indicated in the manuscript.

Conflict of interests: The authors declare that they have no potential conflicting interests.

\section{References:}

Adamson, C., Kanu, O. O., Mehta, A. I., Di, C., Lin, N., Mattox, A. K., \& Bigner, D. D. (2009). Glioblastoma multiforme: a review of where we have been and where we are going. Expert Opinion on Investigational Drugs, 18(8), 1061-1083. https://doi.org/10.1517/13543780903052764

Ahmad, A., Sarkar, S. H., Aboukameel, A., Ali, S., Biersack, B., Seibt, S., ... Sarkar, F. H. (2012). Anticancer action of garcinol in vitro and in vivo is in part mediated through inhibition of STAT3 signaling. Carcinogenesis, 33(12), 2450-2456. https://doi.org/10.1093/carcin/bgs290

Ahmad, A., Wang, Z., Ali, R., In, M. ', Maitah, Y., Kong, D., ... Sarkar, F. H. (2010). ApoptosisInducing Effect of Garcinol Is Mediated by NF-kB Signaling in Breast Cancer Cells. J. Cell. Biochem, 109, 1134-1141. https://doi.org/10.1002/jcb.22492

Arshad, L., Haque, M.A., Abbas Bukhari, S.N., Jantan, I. (2017). An overview of structure-activity relationship studies of curcumin analogs as antioxidant and anti-inflammatory agents. Future Med Chem. 9(6):605-626. doi: 10.4155/fmc-2016-0223. 
435

436

437

438

439

440

441

442

443

444

445

446

447

448

449

450

451

452

453

454

455

456

457

458

459

460

461

462

463

464

465

466

467

468

469

470

471

472

473

474

475

476

477

478

479

480

481

482

483

484

485

486

Beier, D., Schulz, J. B., \& Beier, C. P. (2011). Chemoresistance of glioblastoma cancer stem cells - much more complex than expected. Molecular Cancer, 10(1), 128. https://doi.org/10.1186/1476-4598-10128

Bell, E.H., Zhang, P., Fisher, B.J, et al. Association of MGMT Promoter Methylation Status With Survival Outcomes in Patients With High-Risk Glioma Treated With Radiotherapy and Temozolomide: An Analysis From the NRG Oncology/RTOG 0424 Trial. (2018). JAMA Oncol. 4(10):1405-1409. doi:10.1001/jamaoncol.2018.1977

Bhaskaran, V., Nowicki, M.O., Idriss, M., Jimenez, M.A., Lugli, G., Hayes, J.L., et al. (2019). The functional synergism of microRNA clustering provides therapeutically relevant epigenetic interference in glioblastoma. Nat Comm.10(1), 442. doi: 10.1038/s41467-019-08390-z.

Cao, S., Wang, C., Zheng, Q., Qiao, Y., Xu, K., Jiang, T., \& Wu, A. (2011). STAT5 regulates glioma cell invasion by pathways dependent and independent of STAT5 DNA binding. Neuroscience Letters, 487(2), 228-233. https://doi.org/10.1016/J.NEULET.2010.10.028

Carro, Maria stella; Lim, Wei; Alvarez, Mariano; Bollo, Robert; Zhao, Xudong; Snyder, Evan; Sulman, Erik; Anne, Sandrine; Doetsch, Fiona; Colman, Howard; Lasorella, Anna; Aldape, Ken; Califano, Andrea; Iavarone, A. (2010). The transcriptional network for mesenchymal transformation of brain tumors. Nature, 7279(463), 318-325. https://doi.org/10.1097/CCM.0b013e31823da96d.Hydrogen

Cheng, C.-C., Shi, L.-H., Wang, X.-J., Wang, S.-X., Wan, X.-Q., Liu, S.-R., ... Ding, Y. (2018). Stat3/Oct4/c-Myc signal circuit for regulating stemness-mediated doxorubicin resistance of triplenegative breast cancer cells and inhibitory effects of WP1066. International Journal of Oncology, 53(1), 339-348. https://doi.org/10.3892/ijo.2018.4399

Colak, S., \& Medema, J. P. (2014). Cancer stem cells - Important players in tumor therapy resistance. FEBS Journal, 281(21), 4779-4791. https://doi.org/10.1111/febs.13023

Do, D. V., Ueda, J., Messerschmidt, D. M., Lorthongpanich, C., Zhou, Y., Feng, B., ... Fu, X.-Y. (2013). A genetic and developmental pathway from STAT3 to the OCT4-NANOG circuit is essential for maintenance of ICM lineages in vivo. Genes $\mathcal{E}$ Development, 27(12), 1378-1390. https://doi.org/10.1101/gad.221176.113

Fan, Q. W., Cheng, C. K., Gustafson, W. C., Charron, E., Zipper, P., Wong, R. A., ... Weiss, W. A. (2013). EGFR Phosphorylates Tumor-Derived EGFRvIII Driving STAT3/5 and Progression in Glioblastoma. Cancer Cell, 24(4), 438-449. https://doi.org/10.1016/j.ccr.2013.09.004

Floyd, D., \& Purow, B. (2014). Micro-masters of glioblastoma biology and therapy: increasingly

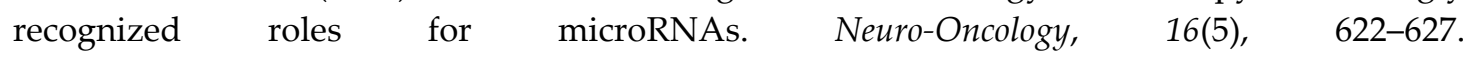
https://doi.org/10.1093/neuonc/nou049

Frank, N. Y., Schatton, T., \& Frank, M. H. (2010). The therapeutic promise of the cancer stem cell concept. Journal of Clinical Investigation, 120(1), 41-50. https://doi.org/10.1172/JCI41004

Ghanem, S., Friedbichler, K., Boudot, C., Bourgeais, J., Gouilleux-Gruart, V., Régnier, A., ... Gouilleux, F. (2017). STAT5A/5B-specific expansion and transformation of hematopoietic stem cells. Blood Cancer Journal, 7(1), e514-e514. https://doi.org/10.1038/bcj.2016.124

Gleixner, K.V., Schneeweiss, M.A., Herrmann, H., Blatt, K., Berger, D., Eisenwort, G., et al. (2016). Combined Targeting of STAT3 and STAT5: A Novel Approach to Overome Drug Resistance in Ph+ Cml. Blood 128: 4241

Guryanova, O. A., Wu, Q., Cheng, L., Lathia, J. D., Huang, Z., Yang, J., ... Bao, S. (2011). Nonreceptor Tyrosine Kinase BMX Maintains Self-Renewal and Tumorigenic Potential of Glioblastoma Stem Cells by Activating STAT3. Cancer Cell, 19(4), 498-511. https://doi.org/10.1016/j.ccr.2011.03.004

Hegi, M. E., Diserens, A.-C., Gorlia, T., Hamou, M.-F., de Tribolet, N., Weller, M., ... Stupp, R. (2005). MGMT Gene Silencing and Benefit from Temozolomide in Glioblastoma. New England Journal of Medicine, 352(10), 997-1003. https://doi.org/10.1056/NEJMoa043331

Ho, K.-H., Chen, P.-H., Hsi, E., Shih, C.-M., Chang, W.-C., Cheng, C.-H., .. Chen, K.-C. (2017). Identification of IGF-1-enhanced cytokine expressions targeted by miR-181d in glioblastomas via an integrative miRNA/mRNA regulatory network analysis. Scientific Reports, 7(1), 732. https://doi.org/10.1038/s41598-017-00826-0 
Hong, J., Kwon, S. J., Sang, S., Ju, J., Zhou, J., Ho, C.-T., ... Yang, C. S. (2007). Effects of garcinol and its derivatives on intestinal cell growth: Inhibitory effects and autoxidation-dependent growthstimulatory effects. Free Radical Biology and Medicine, 42(8), 1211-1221. https://doi.org/10.1016/J.FREERADBIOMED.2007.01.016

Huang, W. C., Kuo, K. T., Adebayo, B. O., Wang, C. H., Chen, Y. J., Jin, K., ... Yeh, C. T. (2018). Garcinol inhibits cancer stem cell-like phenotype via suppression of the Wnt/ $\beta$-catenin/STAT3 axis signalling pathway in human non-small cell lung carcinomas. Journal of Nutritional Biochemistry, 54, 140-150. https://doi.org/10.1016/j.jnutbio.2017.12.008

Jackson, M., Hassiotou, F., \& Nowak, A. (2015). Glioblastoma stem-like cells: at the root of tumor recurrence and a therapeutic target. Carcinogenesis, 36(2), 177-185. https://doi.org/10.1093/carcin/bgu243

Kim, J., Patel, M., Ruzevick, J., Jackson, C., Lim, M., Kim, J. E., ... Lim, M. (2014). STAT3 Activation in Glioblastoma: Biochemical and Therapeutic Implications. Cancers, 6(1), 376-395. https://doi.org/10.3390/cancers6010376

Kreisl, T. N., Kim, L., Moore, K., Duic, P., Royce, C., Stroud, I., ... Fine, H. A. (2009). Phase II trial of single-agent bevacizumab followed by bevacizumab plus irinotecan at tumor progression in recurrent glioblastoma. Journal of Clinical Oncology, 27(5), 740-745. https://doi.org/10.1200/JCO.2008.16.3055

Kwon, S.-J., Kwon, O.-S., Kim, K.-T., Go, Y.-H., Yu, S.-I., Lee, B.-H., ... Cha, H.-J. (2017). Role of MEK partner-1 in cancer stemness through MEK/ERK pathway in cancerous neural stem cells, expressing EGFRviii. Molecular Cancer, 16(1), 140. https://doi.org/10.1186/s12943-017-0703-y

Latha, K., Li, M., Chumbalkar, V., Gururaj, A., Hwang, Y., Dakeng, S., ... Furnari, F. B. (2013). Nuclear EGFRvIII-STAT5b complex contributes to glioblastoma cell survival by direct activation of the Bcl-XL promoter. International Journal of Cancer, 132(3), 509-520. https://doi.org/10.1002/ijc.27690

Li, G., Wei, H., Chen, Z., Lv, S., Yin, C., \& Wang, D. (2009). STAT3 silencing with lentivirus inhibits growth and induces apoptosis and differentiation of U251 cells. Journal of Neuro-Oncology, 91(2), 165-174. https://doi.org/10.1007/s11060-008-9696-0

Matthews, J. R., Sansom, O. J., \& Clarke, A. R. (2011). Absolute requirement for STAT3 function in small-intestine crypt stem cell survival. Cell Death \& Differentiation, 18(12), 1934-1943. https://doi.org/10.1038/cdd.2011.77

Mohanty, S. K., Yagiz, K., Pradhan, D., Luthringer, D. J., Amin, M. B., Alkan, S., \& Cinar, B. (2017). STAT3 and STAT5A are potential therapeutic targets in castration-resistant prostate cancer. Oncotarget, 8(49), 85997-86010. https://doi.org/10.18632/oncotarget.20844

Omuro, A., \& DeAngelis, L. M. (2013). Glioblastoma and other malignant gliomas: A clinical review. JAMA - Journal of the American Medical Association, Vol. 310, pp. 1842-1850. https://doi.org/10.1001/jama.2013.280319

Phi, L. T. H., Sari, I. N., Yang, Y.-G., Lee, S.-H., Jun, N., Kim, K. S., ... Kwon, H. Y. (2018). Cancer Stem Cells (CSCs) in Drug Resistance and their Therapeutic Implications in Cancer Treatment. Stem Cells International, 2018, 1-16. https://doi.org/10.1155/2018/5416923

Pop-Bica, C., Pintea, S., Cojocneanu-Petric, R., Del Sal, G., Piazza, S., Wu, Z.H., et al. (2018). MiR-181 family-specific behaviour in different cancers: a meta-analysis view. Cancer Metastasis Rev. 37(1):17-32. doi: 10.1007/s10555-017-9714-9.

Roos, A., Dhruv, H. D., Peng, S., Inge, L. J., Tuncali, S., Pineda, M., ... Tran, N. L. (2018). EGFRvIIIStat5 Signaling Enhances Glioblastoma Cell Migration and Survival. Molecular Cancer Research, 16(7), 1185-1195. https://doi.org/10.1158/1541-7786.MCR-18-0125

Rossetti, A. O., \& Stupp, R. (2010). Epilepsy in brain tumor patients. Current Opinion in Neurology, 23(6), 603-609. https://doi.org/10.1097/WCO.0b013e32833e996c

Saadat, N., \& Gupta, S. V. (2012). Potential Role of Garcinol as an Anticancer Agent. Journal of Oncology, 2012, 1-8. https://doi.org/10.1155/2012/647206

Lathia, J. D., Mack, S. C., Mulkearns-Hubert, E. E., Valentim, C. L., Rich, J. N. (2015). Cancer stem cells in glioblastoma. Genes Dev, 29(12), 1203-1217. doi:10.1101/gad.261982.115

Stupp, R., Mason, W. P., van den Bent, M. J., Weller, M., Fisher, B., Taphoorn, M. J. B., .. National 
Cancer Institute of Canada Clinical Trials Group. (2005). Radiotherapy plus concomitant and adjuvant temozolomide for glioblastoma. The New England Journal of Medicine, 352(10), 987-996. https://doi.org/10.1056/NEJMoa043330

Toledo-Guzmán, M. E., Bigoni-Ordóñez, G. D., Ibáñez Hernández, M., \& Ortiz-Sánchez, E. (2018). Cancer stem cell impact on clinical oncology. World Journal of Stem Cells, 10(12), 183-195. https://doi.org/10.4252/wjsc.v10.i12.183

Yang, F., Liu, X., Liu, Y., Liu, Y., Zhang, C., Wang, Z., ... Wang, Y. (2017). miR-181d/MALT1 regulatory axis attenuates mesenchymal phenotype through NF- $\kappa$ B pathways in glioblastoma. Cancer Letters, 396, 1-9. https://doi.org/10.1016/j.canlet.2017.03.002

Yuan, J., Zhang, F., Niu, R. (2015). Multiple regulation pathways and pivotal biological functions of STAT3 in cancer. Sci Rep. 5:17663. doi: 10.1038/srep17663.

Yue, P., \& Turkson, J. (2009). Targeting STAT3 in cancer: how successful are we? Expert Opinion on Investigational Drugs, 18(1), 45-56. https://doi.org/10.1517/13543780802565791

Zhang, W., Zhang, J., Hoadley, K., Kushwaha, D., Ramakrishnan, V., Li, S., ... Chen, C. C. (2012). miR-181d: a predictive glioblastoma biomarker that downregulates MGMT expression. NeuroOncology, 14(6), 712-719. https://doi.org/10.1093/neuonc/nos089

\section{Figure Legends}

A

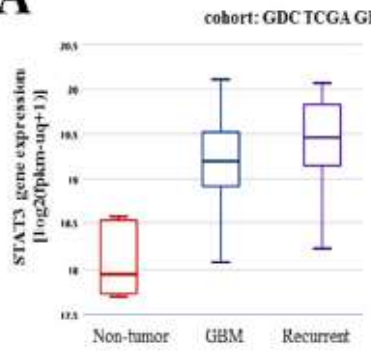

B
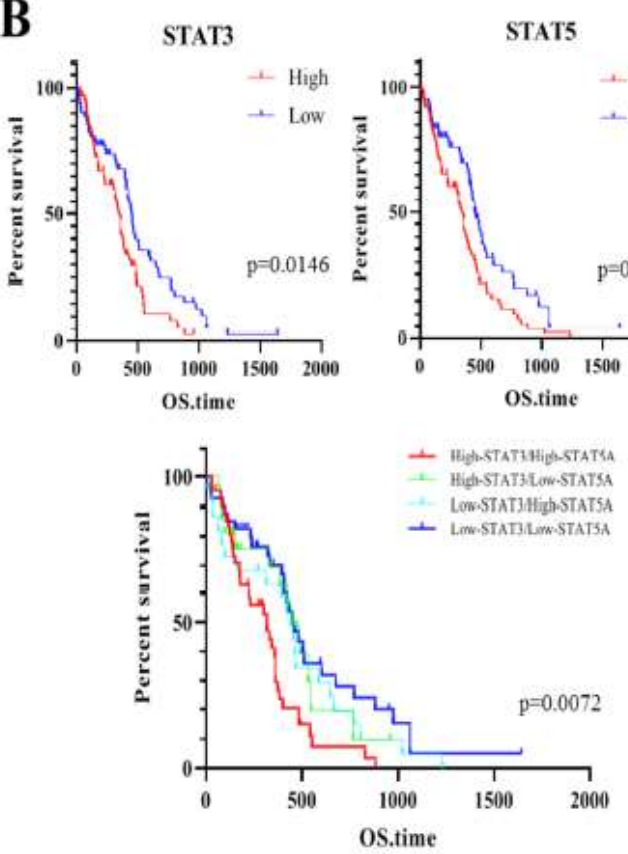

C

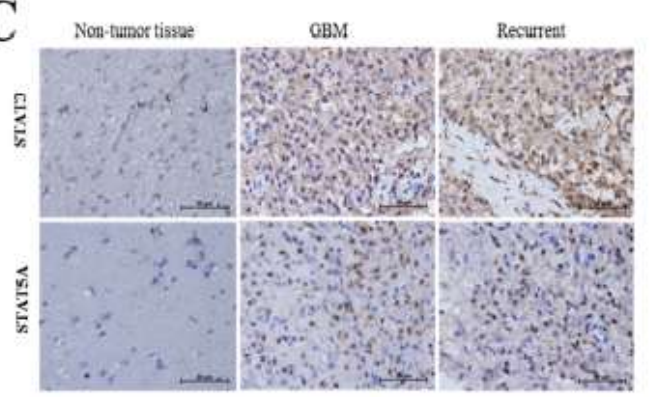

D
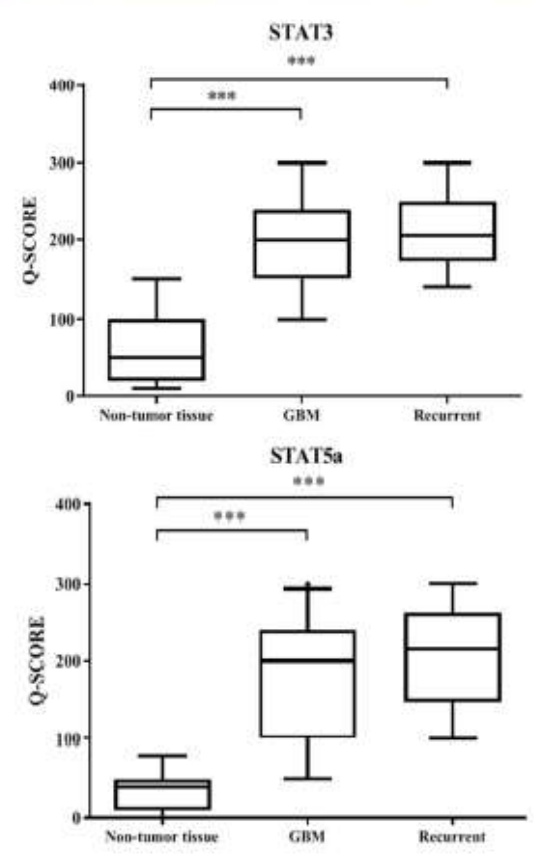

Figure 1. STAT-3 and STAT-5 are highly expressed in primary and recurrent glioblastoma, and their expression negatively correlates with overall survival rates. (A) Graphical representation of the differential expression of STAT3 and STAT5A in primary GBM, recurrent GBM or normal brain tissues from the GDC TCGA-GBM cohort. (B) Kaplan-Meier plots of the effect of differential STAT3 
or STAT5A expression on OS of patients with GBM. (C) Representative IHC images showing the differential expression of STAT3 and STAT5A proteins in primary GBM, recurrent GBM or normal brain tissues. (D) Graphical representation of the differential expression of STAT3 and STAT5A proteins in primary GBM, recurrent GBM or normal brain tissues. ${ }^{*} p<0.05,{ }^{* *} p<0.01,{ }^{* * *} p<0.001$; OS, overall survival.

A

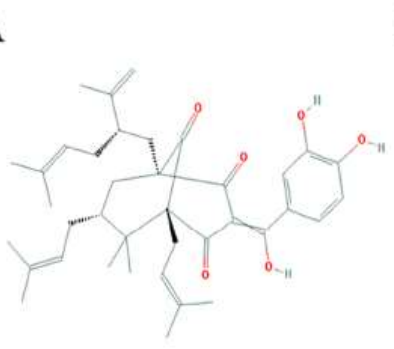

Garcinol $\mathrm{C}_{38} \mathrm{H}_{50} \mathrm{O}_{6}$ MW : $602.812 \mathrm{~g} / \mathrm{mol}$

B

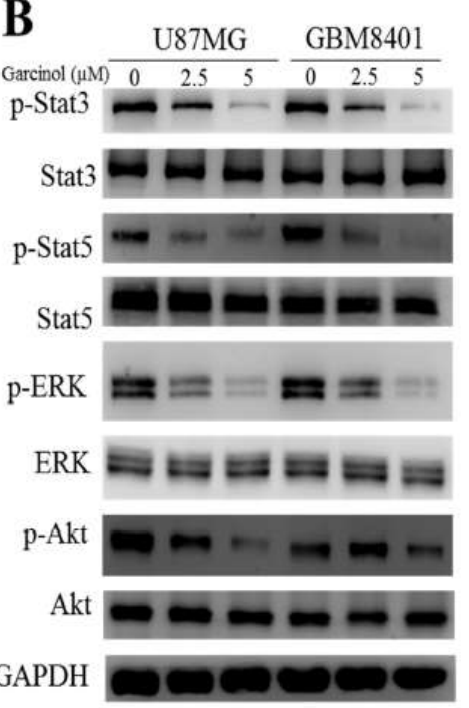

C

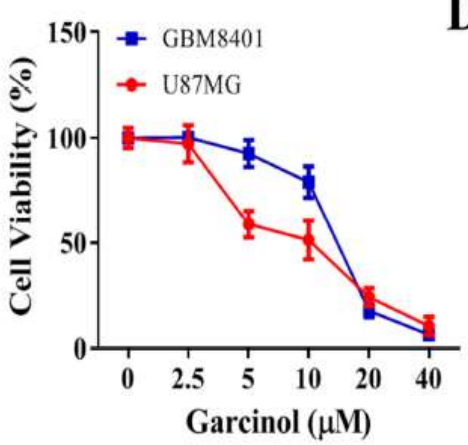

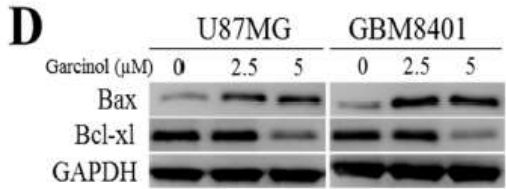

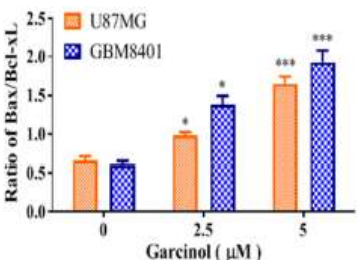

E $\frac{\text { U87MG }}{0 \text { GBM8401 }}$
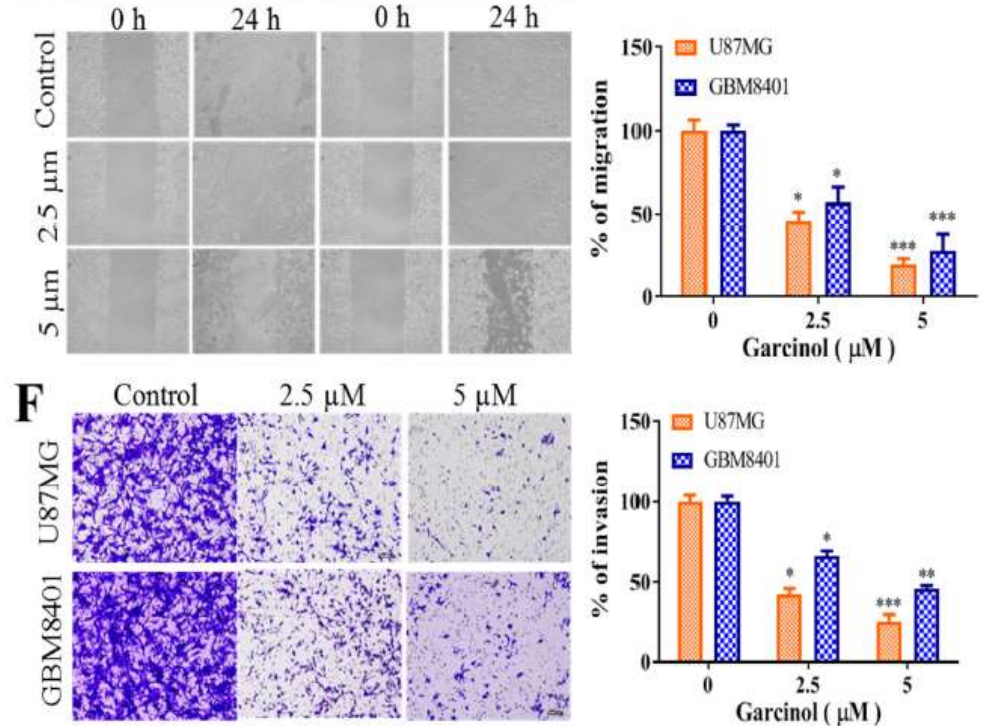

Figure 2. Garcinol significantly inhibits GBM cell viability and oncogenicity through induction of STAT3/5A signaling and enhanced apoptosis. (A) Chemical structure of garcinol with molecular formula $\mathrm{C}_{38} \mathrm{H}_{50} \mathrm{O}_{6}$ and molecular weight $602.80 \mathrm{~g} / \mathrm{mol}$. (B) Representative western blot photo-images of the effect of $2.5 \mu \mathrm{M}-5 \mu \mathrm{M}$ on the expression of p-STAT3, STAT3, p-STAT5, STAT5, p-ERK, ERK, p-AKT, and AKT proteins in GBM8401 or U87MG cells. (C) Graphical representation of the effect of $2.5 \mu \mathrm{M}-40 \mu \mathrm{M}$ on the viability of GBM8401 or U87MG cells. (D) Representative western-blot photoimages showing the effect of $2.5 \mu \mathrm{M}-5 \mu \mathrm{M}$ on the expression of Bax and Bcl-xL proteins in GBM8401 or U87MG cells. Representative photo-images (upper) and graphical representation (lower) of the effect of $2.5 \mu \mathrm{M}$ or $5 \mu \mathrm{M}$ on the (E) migration and (F) invasion of GBM8401 or U87MG cells. ${ }^{*} \mathrm{p}<0.05$, ${ }^{* *} \mathrm{p}<0.01,{ }^{* * *} \mathrm{p}<0.001 ; \mathrm{GAPDH}$ is loading control. 

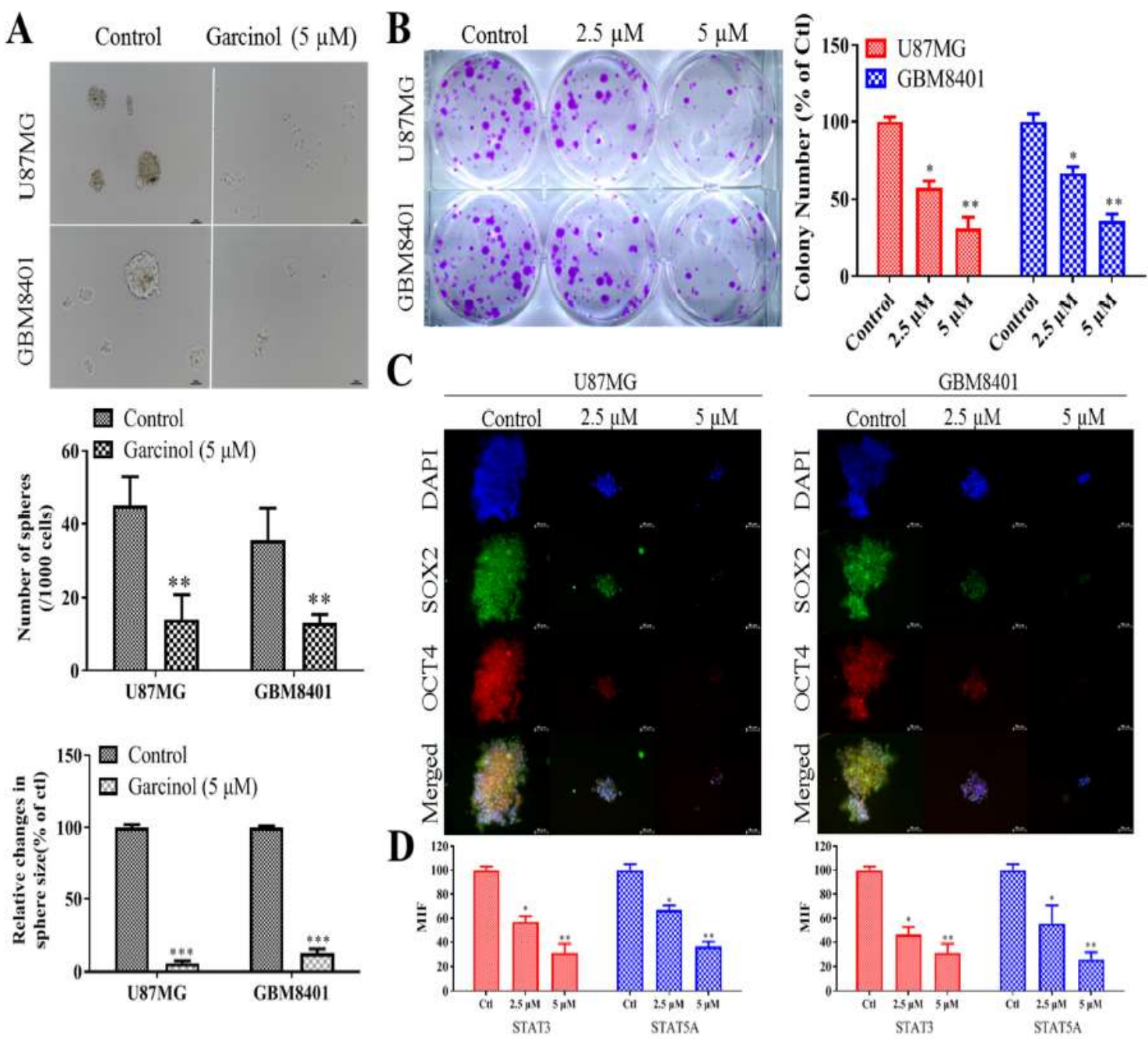

Figure 3. Garcinol negatively impacts GBM stem cell-like phenotypes. (A) Representative photoimages (upper) and histograms (middle, lower) of the effect of $5 \mu \mathrm{M}$ on the number and size of tumorspheres formed by U87MG or GBM8401 cells. (B) Representative images of the effect of $2 \mu \mathrm{M}$ or $5 \mu \mathrm{M}$ on the number of colonies formed by U87MG or GBM8401 cells. (C) Representative photoimages showing the effect of $2 \mu \mathrm{M}$ or $5 \mu \mathrm{M}$ on the tumorsphere size and sub-cellular localization of SOX2 and OCT4 proteins in U87MG or GBM8401 cells. (D) Graphs showing how $2 \mu \mathrm{M}$ or $5 \mu \mathrm{M}$ affect the MIF of STAT3 and STAT5A in U87MG or GBM8401 cells. ${ }^{*} p<0.05,{ }^{* *} p<0.01,{ }^{* * *} p<0.001 ;$ MIF, 


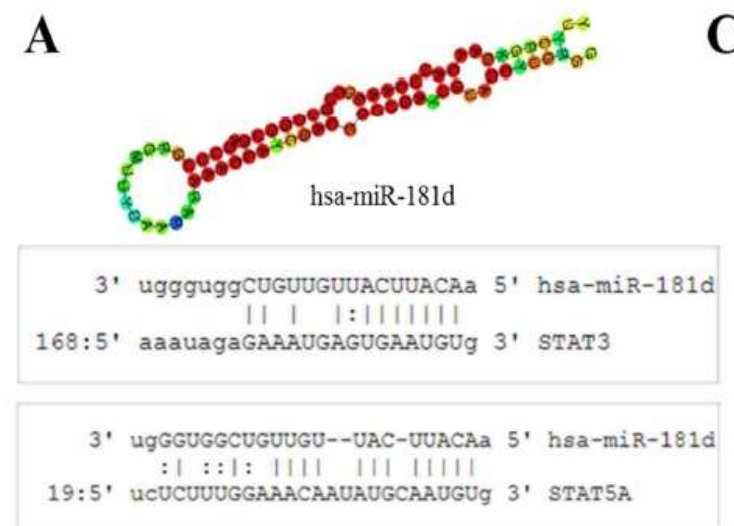

B

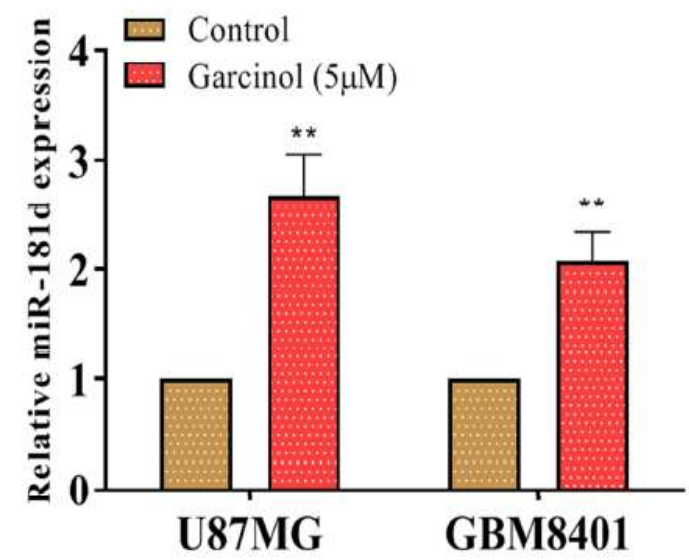

C

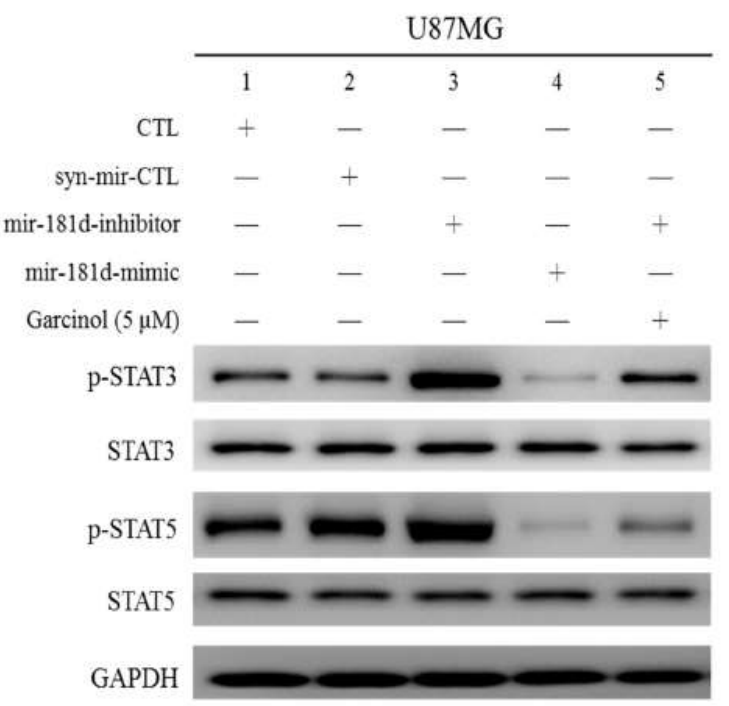

Figure 4. Garcinol increases the expression of hsa-miR181d, which has inhibitory effects on STAT3 and STAT5 expression. (A) 2-dimensional image of hsa-miR-181d (upper) and images showing the complementary sequence alignment of hsa-miR-181d with STAT3 (middle) or STAT5A (lower). The mirSVR and PhastCons scores are indicated. (B) Histograms of the effect of $5 \mu \mathrm{M}$ on U87MG or GBM8401 cells. (C) Representative western-blot photo-images comparing the effect of Syn-mir-CTL, mir-181d-inhibitor, mir-181d-mimic, or garcinol on the expression level of p-STAT3, STAT3, p-STAT5, and STAT5 in U87MG or GBM8401 cells. ${ }^{*} \mathrm{p}<0.05,{ }^{* *} \mathrm{p}<0.01,{ }^{* * *} \mathrm{p}<0.001$; GAPDH is loading control. 


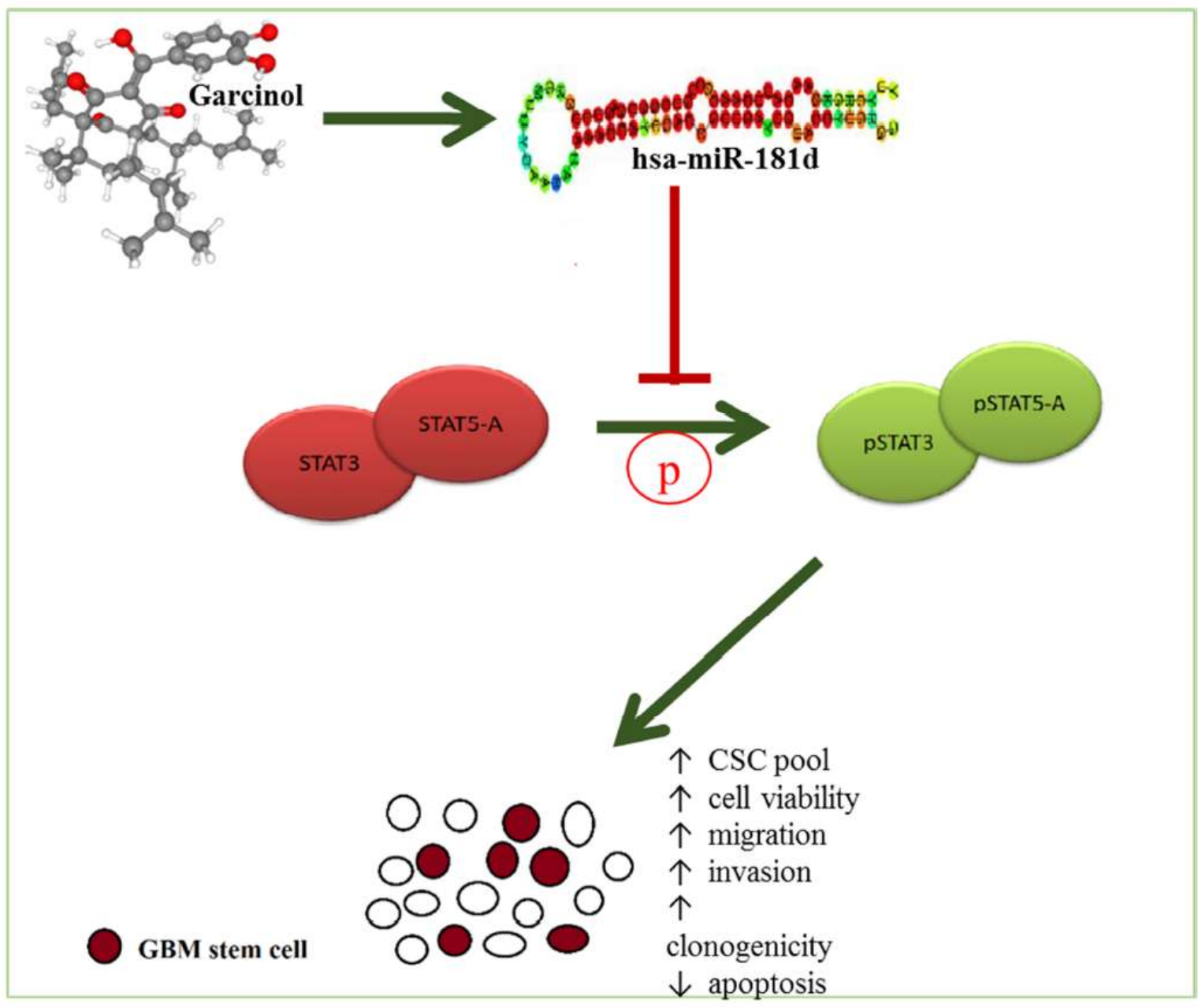

Figure 5. Pictorial abstract showing how activation of STAT3/5A in GBM is inversely correlated with suppressed has-miR-181d expression, and that garcinol-induced upregulation of hsa-miR$181 \mathrm{~d} /$ STAT3 and hsa-miR-181d/5A ratios mediate the anti-GBM-SCs effect of garcinol in STAT3/5Aaddicted GBM. 